\title{
Nutritional advice for prevention of acute pancreatitis: review of current opinion
}

\author{
This article was published in the following Dove Press journal: \\ Nutrition and Dietary Supplements \\ II December 2012 \\ Number of times this article has been viewed
}

\author{
Mark E Lowe \\ Wednesday A Sevilla \\ Division of Pediatric \\ Gastroenterology, Hepatology and \\ Nutrition, Children's Hospital of \\ Pittsburgh of UPMC, Pittsburgh, \\ PA, USA
}

\begin{abstract}
Pancreatitis is inflammation of the pancreas that can be acute and self-limiting or, in a small percentage of patients, recurrent. Patients with recurrent episodes of acute pancreatitis (RAP) often progress to chronic pancreatitis. Pancreatitis in all forms causes significant economic and social burdens. Prevention of RAP may decrease those burdens and halt progression to chronic disease. Unfortunately, no therapy has proven effective at altering the course of RAP. While enteral nutritional therapy plays an important role in the treatment of acute pancreatitis during episodes, nutritional advice provided to patients in an attempt to prevent recurrent episodes has not proven effective in most cases. Discontinuing alcohol consumption and treating dyslipidemia with diet and medication can help patients with these issues. In patients whose pancreatitis is associated with celiac disease or eosinophilic gastroenteritis, a gluten-free diet and avoidance of food allergens can be effective in stopping RAP. Advice to take pancreatic enzyme replacement therapy, lose weight, control diabetes, decrease dietary sucrose, decrease dietary fat or avoid monosodium glutamate has little to no evidence of efficacy. Some studies suggest that an antioxidant cocktail may decrease the frequency of RAP and the intensity of chronic pain, but the evidence is weak. Nutritional therapy may have a role in the treatment of patients with RAP. At present, there are no clear guidelines for nutritional advice to give these patients. More studies are needed to identify nutritional interventions that will benefit patients with RAP.
\end{abstract}

Keywords: pancreatitis, nutrition, pancreatic enzyme replacement therapy, antioxidants, herbal supplements

\section{Introduction}

Pancreatitis is an inflammatory disorder of the pancreas. The inflammatory process can localize in the pancreas or spread to tissues surrounding the pancreas or to other organs. ${ }^{1}$ Most cases of pancreatitis are acute, and patients recover from the attack with no evidence of clinical, histological, or functional changes. A small subset of patients has recurrent episodes of acute pancreatitis. Recurrent acute pancreatitis (RAP) is defined by relapsing episodes of pancreatitis with morphologic restitution and preservation of function once symptoms have resolved. Acute pancreatitis and recurrent acute pancreatitis can develop into chronic pancreatitis. Chronic pancreatitis is defined as persistent inflammation in the pancreas with irreversible morphologic or functional pancreatic changes. $^{2}$

Pancreatitis lacks effective therapy and presents a significant health and economic impact. In 2004, there were 475,000 recorded ambulatory care visits for pancreatitis. These rates have been steadily increasing since $1979 .{ }^{3}$ Total costs for management of pancreatitis 
cases were estimated at $\$ 3.7$ billion for 2004 . Between $10 \%$ and $20 \%$ of patients have recurrent episodes of acute pancreatitis. Some, perhaps all, of these patients eventually develop chronic pancreatitis, a disease that adds considerably to the health and economic impact of pancreatic disorders. At present, therapy for acute pancreatitis and chronic pancreatitis is supportive. No therapy exists to prevent recurrent episodes of acute pancreatitis or progression to chronic pancreatitis. Effective therapy for these disorders would dramatically change the lives of patients and impact significantly on the economic burden of acute recurrent pancreatitis and chronic pancreatitis. Importantly, effective therapy should stop progression to chronic pancreatitis and its complications, such as debilitating pain, maldigestion, malnutrition, and diabetes mellitus.

In this article, we review concepts in nutritional therapy to prevent pancreatitis. We briefly discuss the nutritional pathophysiology and nutritional management of acute pancreatitis. Lastly, we summarize available data on nutritional therapy to prevent recurrent pancreatitis episodes.

\section{Nutritional pathophysiology in pancreatitis}

Like most disease states, patients with pancreatitis have a negative energy balance. ${ }^{1}$ They have increased caloric expenditure from inflammation and decreased intake due to abdominal pain and anorexia. There are signs of hyperdynamic changes and, in some patients, elevated resting energy expenditure. ${ }^{4,5}$ Also present is a higher rate of net protein catabolism with a decrease in the serum amino acid pool. Carbohydrate metabolism can be impaired due to higher levels of stress hormones with a subsequent need for control with exogenous insulin. Carbohydrate metabolism is further complicated when pancreatitis progresses to its chronic form when irreversible injury to the pancreatic islets occurs. ${ }^{2}$ Less frequently, hyperlipidemia in the form of hypertriglyceridemia is seen from increased lipolysis and lipid oxidation, with concordant poor clearance from serum. ${ }^{1}$

Electrolyte and micronutrient deficiencies are observed. Hypocalcemia and hypomagnesemia can occur even in the first episode of acute pancreatitis. ${ }^{6,7}$ Patients with longstanding excessive alcohol intake may have thiamine and folate deficiencies in addition to protein-calorie malnutrition. Patients with chronic pancreatitis can have deficiency of fat-soluble vitamins, particularly vitamins D and K. Vitamin B12 deficiency is observed in patients with pancreatic insufficiency because pancreatic proteases degrade R-binding protein allowing cobalamin to transfer to intrinsic factor. The complex is then absorbed in the distal ileum. ${ }^{1}$
The main tenet of nutritional management in pancreatitis is to meet the energy needs of the patient through appropriate calorie administration. This is particularly important in cases of acute and recurrent acute pancreatitis because it will likely reduce complications and decrease hospital stays. ${ }^{8}$

\section{Nutritional therapy and supplements in acute pancreatitis}

The majority of patients with pancreatitis have a good prognosis, but a small number will develop severe illness that requires intensive care and intensive nutritional support. ${ }^{9}$ For many years, parenteral feeding was considered the natural choice for nutrition in critically ill patients with acute pancreatitis. Current evidence favors enteral tube feeding. Enteral tube feeding has fewer complications and is less expensive than parenteral nutrition and improves outcomes in critically ill patients. ${ }^{10,11}$ Enteral tube feeds lower infection rates, reduce surgical interventions, decrease length of hospital stay, decrease organ failure, and decrease overall mortality rates ${ }^{11,12}$ (see Table 1 ).

There are currently no clear guidelines on the best mode of enteral feeding administration, ie, nasogastric or nasojejunal. Theoretical arguments have been offered for the use of nasojejunal feeding. ${ }^{13}$ Placing the tube $40-60 \mathrm{~cm}$ past the ligament of Treitz decreases pancreatic secretions compared with duodenal feeds and stimulates glucagon-like peptide 1 and peptide YY, two components in the activation of the "ileal brake" that inhibits pancreatic secretion. Critically ill patients with pancreatitis tolerate nasojejunal feeds. Occasionally, placement of the feeding tube in the distal jejunum can be difficult. ${ }^{14}$ Other investigators recommend nasogastric tube feeds and have demonstrated excellent safety and tolerance in head-to-head comparisons with nasojejunal feeding. ${ }^{15,16}$ An ongoing study comparing the two methods may provide additional guidance for the choice of tube placement. Once the tube is placed, the use of semielemental or polymeric formula does not influence the risk of complications, feeding intolerance, or mortality. ${ }^{17}$

Table I Benefits of enteral feeding in management of acute pancreatitis

Few infectious complications

Lower cost

Reduces length of hospital stay

Reduces surgical intervention

Decreases incidence of organ failure

Decreases overall mortality rate 
In mild cases of acute pancreatitis, current international guidelines recommend gradual advancement of diet as tolerated, usually over 3-4 days. ${ }^{18}$ Resumption of feeding occurred earlier in patients who self-selected when to eat compared with those guided by lipase levels with no differences in caloric intake and pain symptoms. ${ }^{19}$ Thus, normalization of serum lipase should not guide when to start enteral nutrition. Even a low caloric intake provides more benefit than parenteral nutrition and bowel rest in terms of safety and cost. ${ }^{20}$ Increased pain is the most important adverse effect of refeeding. ${ }^{21}$ Pain increases in about $10 \%$ of patients. Interrupting feeds for a brief period of time is generally all that is needed before these patients tolerate feeding.

The approach to restarting feeds has changed from gradual advancement from clear liquids to a solid diet to allowing a solid diet from the beginning. A prospective, randomized trial showed that a low-fat (35 $\mathrm{g}$ fat per day) solid diet was as well tolerated as a clear liquid diet. ${ }^{22}$ Although the low-fat solid diet did not shorten hospital stay, it provided more calories and fat on resumption of diet. The authors concluded that a clear liquid diet and low-fat solid diet were both acceptable when restarting an oral diet in cases of mild acute pancreatitis. Another study compared the tolerability of a hypocaloric liquid diet, a hypocaloric soft diet, and a full caloric solid diet (45 $\mathrm{g}$ fat per day) in patients recovering from mild acute pancreatitis. The full caloric solid diet was as well tolerated as the other diets and resulted in a shorter hospital stay. ${ }^{23}$ The authors concluded that patients with mild acute pancreatitis can eat a full solid diet early in the course of the episode without experiencing harmful effects. No one has yet tested the tolerability and efficacy of a diet containing higher amounts of fat.

The role of other nutritional supplements in treating acute pancreatitis is unclear, and only a few studies have addressed their use. Because reactive oxidative species may play an important role in the pathophysiology of pancreatitis, several studies have addressed the use of various antioxidants. Trials of glutamine, vitamin C, S-adenosyl methionine, selenium, and $\mathrm{N}$-acetylcysteine, either as monotherapy or combination therapy, showed no significant differences in outcome parameters, such as disease severity score, hospitalization, or mortality. ${ }^{24} \mathrm{~A}$ recent review of antioxidant therapy concluded that there are no distinct guidelines for the effective use of antioxidants and further studies are required. ${ }^{25}$ An initial study of probiotics given in conjunction with enteral nutrition suggested probiotics decreased the incidence of abdominal abscesses and decreased the length of stay, but a subsequent large trial showed statistically significant higher mortality precluding routine use of probiotics in severe acute pancreatitis. ${ }^{26,27}$ Other nutrient substrates such as glutamine, omega-3 fatty acids, and nucleotides (also known as immunonutrition supplements) have been explored, but no differences in clinical outcomes were identified. ${ }^{28}$

\section{Nutritional modifications to prevent recurrent acute pancreatitis}

Most patients with acute pancreatitis have one episode while others may develop recurrent episodes. In some of these patients, an etiology can be identified and treated. In the majority, no etiology is identified or they have a genetic risk factor for which there is no treatment. Patients are often given nutritional advice in the hope that the frequency or severity of episodes will decrease. In this section, we discuss available evidence for various nutritional therapies and their influence on outcome (Table 2).

\section{Alcohol}

A population study of pancreatitis showed that alcohol consumption contributed to half of the cases of chronic pancreatitis. ${ }^{29}$ Patients in this etiologic group were more likely to be male and younger (median age 51.5 years). Importantly, patients with alcohol-related chronic pancreatitis have more complicated disease and have a significantly shortened lifespan. The risk is associated with amount of alcohol intake. Consumption of more than five drinks per day increased the hazard ratio to more than $3 .{ }^{30}$ Progression to chronic pancreatitis is more likely to occur in patients with a history of recurrent acute pancreatitis and who continue alcohol consumption. A well designed, randomized trial

Table 2 Nutritional interventions in recurrent episodes of acute pancreatitis and chronic pancreatitis

\begin{tabular}{|c|c|}
\hline Intervention & Benefits \\
\hline Alcohol abstinence & $\begin{array}{l}\text { Can decrease risk of recurrence; } \\
\text { long-term intervention needed }\end{array}$ \\
\hline Weight loss & $\begin{array}{l}\text { Obesity may increase risk; no data if } \\
\text { weight reduction can decrease risk }\end{array}$ \\
\hline Treatment of dyslipidemia & $\begin{array}{l}\text { Control of triglyceride levels can } \\
\text { decrease risk }\end{array}$ \\
\hline Treatment of diabetes & $\begin{array}{l}\text { Aggressive control with medications } \\
\text { can decrease risk }\end{array}$ \\
\hline Low-fat diet & No evidence for preventing recurrence \\
\hline Limitation of sucrose & Animal studies only; no clinical studies \\
\hline Increasing dietary fish oil & Animal studies only; no clinical studies \\
\hline $\begin{array}{l}\text { Food sensitivity avoidance } \\
\text { (ie, gluten sensitivity and } \\
\text { food allergy) }\end{array}$ & $\begin{array}{l}\text { Avoidance can prevent recurrence if } \\
\text { causality has been established }\end{array}$ \\
\hline $\begin{array}{l}\text { Food additives } \\
\text { (ie, monosodium glutamate) }\end{array}$ & Animal studies only; no clinical studies \\
\hline
\end{tabular}


looked at outcome and recurrence rates after a first attack of alcohol-associated pancreatitis. ${ }^{31}$ Patients who were counseled about the importance of alcohol abstinence in multiple sessions over time were less likely to have another episode of pancreatitis and those who did have subsequent episodes had fewer recurrences. The study provided evidence that aggressive counseling and periodic follow-up with reinforcement can effectively change the pattern of alcohol consumption and alter the recurrence of pancreatitis.

\section{Obesity}

Obesity is considered a low-grade inflammatory state, and is a risk factor for the development of acute pancreatitis, particularly in association with high alcohol consumption. ${ }^{32,33}$ Obesity is also a risk factor for more severe disease. Patients with obesity had more systemic (ie, organ failure) and local complications such as abscess formation, necrosis, and pseudocysts. ${ }^{33}$ A recent study demonstrated a direct association of increased intrapancreatic adipocyte volume and increased body mass index with severe acute pancreatitis. ${ }^{34}$ The mechanism is not yet defined. Intra-abdominal and visceral fat appear to produce significantly more inflammatory mediators and that increase may lead to more complications during acute pancreatitis. ${ }^{35,36}$ In an experimental model of adipocyte-mediated acinar cell injury, unsaturated fatty acids mediate injury in the pancreas and, perhaps, in remote organs. ${ }^{34}$ Currently, no evidence links obesity to recurrent attacks of pancreatitis, and no studies have explored whether weight reduction after an initial episode of acute pancreatitis decreases the likelihood of future episodes. Still, weight reduction is good health advice and patients should be counseled about weight loss and referred to a weight management program.

\section{Dyslipidemia}

Pancreatitis due to hypertriglyceridemia, whether acute or genetic, is rare. ${ }^{37} \mathrm{~A}$ retrospective cohort study of patients referred to a specialty lipid clinic showed that hypertriglyceridemia as a primary cause for acute pancreatitis was unlikely to occur unless triglycerides level were higher than $20 \mathrm{mM}(1772 \mathrm{mg} / \mathrm{dL})$ at the time of presentation. ${ }^{38}$ In a population study of 230 admissions for pancreatitis, isolated dyslipidemia was detected in a small percentage (10\%) and mortality rates were similar in normolipidemic and dyslipidemic patients. It was also observed that adverse outcomes in patients with dyslipidemia were seen in hypertriglyceridemia, even though past studies did not see this relationship. ${ }^{39,40}$ In cases of acquired hypertriglyceridemia, patients with a pre-existing lipid abnormality can develop pancreatitis in the setting of a secondary factor such as poorly controlled diabetes, alcohol use, or intake of medication such as estrogens and beta-blockers. ${ }^{37}$ Control of triglyceride levels can prevent additional attacks of pancreatitis, specifically if levels are less than $1000 \mathrm{mg} / \mathrm{dL} .^{9,37}$

Levels of high-density lipoprotein (HDL) during acute pancreatitis are low and directly associated with the severity of disease. ${ }^{40}$ Elevation of triglycerides is associated with low HDL levels. The theory proposed is that the inflammatory state in acute pancreatitis increases triglyceride levels and suppresses HDL levels. Bugdaci et al suggested two possible pathways. ${ }^{40}$ First, they found that patients with acute pancreatitis had mild elevation of thyroid-stimulating hormone, possibly due to the systemic inflammatory state in acute pancreatitis. Transient elevation of thyroid-stimulating hormone could increase the level of triglycerides. Second, they proposed that the low HDL levels reduce lipoprotein lipase activity, leading to decreased degradation of triglycerides. ${ }^{40}$ Treatment of dyslipidemia via elevation of HDL levels may contribute to recovery from an acute attack through an anti-inflammatory and antioxidant effect. ${ }^{40}$ There are no published data investigating whether any particular HDL level can prevent future attacks.

\section{Diabetes}

Four recent population studies have shown that patients with type 2 diabetes mellitus are at increased risk for developing acute pancreatitis. ${ }^{41-44}$ In a large cohort of Taiwanese patients, the risk for acute pancreatitis in a patient with type 2 diabetes mellitus was 1.95 -fold greater compared with nondiabetics. ${ }^{41}$ Alcoholism and hepatitis $\mathrm{C}$ infection conferred additional risk for this population. From a cohort of patients in the UK from the General Practice Research Database, the hazard ratio was 2.89 compared with nondiabetics. ${ }^{42}$

Treatment of diabetes with the use of drugs (other than insulin) can decrease the risk for pancreatitis by $37 \%-56 \%$, as found in the population study by Lai et al. ${ }^{41}$ Another cohort study through The Health Improvement Network in the United Kingdom showed that patients with type 2 diabetes mellitus who used insulin and metformin may decrease their risk for pancreatitis. ${ }^{43}$ There are no studies exploring the efficacy of long-term glycemic control or weight loss in preventing acute pancreatitis in these patients.

\section{Dietary composition in recurrent acute pancreatitis and chronic pancreatitis}

Patients with a single episode of acute pancreatitis or with RAP are frequently advised to maintain a low-fat diet. 
This practice pattern is strongly influenced by custom. The advice is based on two theoretical advantages of a low-fat diet. The first is based on the observation that less dietary fat results in decreased release of cholecystokinin. The lower release of cholecystokinin was expected to limit the adverse effects of cholecystokinin such as contraction of the gallbladder and delay of gastric emptying, either of which might influence symptoms in patients with pancreatitis. In practice, even low-fat diets cause gallbladder contractions and gastric emptying is about the same when low-fat and high-fat meals are compared. The second potential advantage of low fat rests on the assumption that pancreatic stimulation is detrimental in patients with pancreatitis and the observation that lipids are the strongest stimulant to pancreatic secretion. ${ }^{45}$ While chronic intake of a high-fat diet ( $40 \%$ of calories as fat) increases pancreatic secretion about two-fold over a low-fat diet ( $10 \%$ of calories as fat), pancreatic stimulation is still robust with a low-fat diet. Additionally, both amino acids and carbohydrates stimulate pancreatic secretions significantly. Furthermore, pancreatic secretion is affected by caloric content and physical properties of the meal as well as nutrient content. Thus, the physiological response to a meal is complex and often unpredictable, particularly in an individual patient. Importantly, there are no studies examining the effect of dietary fat levels on outcome in acute pancreatitis or on preventing reoccurrences of acute pancreatitis.

Animal models indicate that dietary composition may contribute to the development of acute pancreatitis. Rats fed a diet high in sucrose ( $40 \%$, containing $20 \%$ fructose) showed pancreatic hyalinization with focal infiltrates compared to rats fed an isocaloric starch diet. ${ }^{46}$ Immunostains of pancreatic tissue showed a mild islet cell injury in the treatment group. This led the authors to conclude that sucrose can induce pancreatic inflammation, although the mechanism is not quite clear and whether this can lead to acute pancreatitis is not known. Additionally, mice fed a diet rich in fish oils had decreased levels of adipokines, inflammatory components seen in obesity that can contribute to the development of worse outcomes in acute pancreatitis. ${ }^{47}$ Similar studies have not been done in humans. There is no evidence that limiting sucrose or increasing intake of fish oil can prevent subsequent episodes of acute pancreatitis.

Although rare, there are case reports that food allergies can present with RAP. ${ }^{48,49}$ The proposed hypothesis is that inflammation, likely eosinophilic gastroenteritis, caused by hypersensitivity to a particular food causes edema and obstruction of the ampulla of Vater, leading to bile reflux into the pancreatic ducts, and resulting in pancreatitis. ${ }^{48-50}$
Symptoms of pancreatitis are observed to occur a few hours after ingestion of the provoking food and can be accompanied by signs of anaphylaxis, such as urticaria, facial flushing, wheezing, headaches, diarrhea, and myalgia. ${ }^{49}$ Avoidance of the offending food substance can prevent future attacks.

Gluten sensitivity is also implicated as a rare cause of idiopathic recurrent pancreatitis. In a study of a population referred for assessment of sphincter of Oddi dysfunction, celiac disease was diagnosed in $7.1 \% .{ }^{51}$ In this subgroup of patients, all had episodes of recurrent abdominal pain or known idiopathic pancreatitis. These patients also had evidence of periampullary inflammation. The authors concluded that celiac disease should be considered as an etiology for recurrent idiopathic pancreatitis. The mainstay of treatment for celiac disease is a gluten-free diet.

The food additive, monosodium glutamate, may cause pancreatic acinar damage. Rats fed monosodium glutamate in their diet showed ultrastructural changes in pancreatic acinar cells and increased acid phosphatase activity. It was suggested that prolonged and continuous intake of monosodium glutamate would induce pancreatic damage. ${ }^{52} \mathrm{~A}$ more recent study reported by Leschenko et al in 2012 found changes similar to acute pancreatitis. The changes included necrotic and degenerative damage to both exocrine and endocrine cells, inflammation with leukocytes and lymphocytes, fibrosis in the interstitium and vascular region, and edema. ${ }^{53}$ The authors concluded that dietary intake of monosodium glutamate should be considered as a potential cause of pancreatitis. There are currently no published data on the effects of other food additives and artificial food dyes on the pancreas.

\section{Pancreatic enzyme replacement}

Pancreatic enzyme replacement therapy (PERT) is prescribed to treat malabsorption, malnutrition, and pain in patients with pancreatic insufficiency. ${ }^{54} \mathrm{~A}$ systematic review of ten trials on the administration of pancreatic enzymes in the management of chronic pancreatitis was completed by Shafiq et al in $2009 .{ }^{55}$ The main objective of their systematic review was to assess whether treatment with PERT caused any changes in pain frequency and intensity and in the use of pain medications, steatorrhea, weight loss, and quality of life. ${ }^{55}$ Each of the studies was a randomized controlled trial with or without blinding. Together the studies included 361 patients. The studies utilized various preparations of pancreatic enzymes (enteric-coated, nonenteric-coated, acid-protected porcine pancreatic enzymes, and microspheres). A variety of comparisons was made, ie, pancreatic enzyme treatment with 
placebo, type of preparation, and frequency of dosing. The follow-up time varied from 5 days to as long as 4 months. The many differences in study design prevented pooling of the results for meta-analysis. The final conclusion was that no clear recommendations could be made for the use of PERT to ameliorate pain, steatorrhea, weight loss, and quality of life. None of the studies addressed prevention of episodes of recurrent acute pancreatitis. However, PERT is still often prescribed for patients with recurrent acute pancreatitis despite the lack of data supporting efficacy. Table 3 summarizes the trials that were included in the analysis by Shafiq et al. ${ }^{55}$

\section{Antioxidants and herbal supplements}

Oxidative stress is considered to play a primary role in the pathophysiology of pancreatitis, especially in the chronic form. Patients with chronic pancreatitis have lower serum levels of antioxidants and higher levels of markers for oxidative stress. ${ }^{66}$ Consequently, patients with chronic pancreatitis may have a higher risk for repeated pancreatic injury from oxidative stress due to depleted stores of antioxidant enzymes and concurrent inadequate dietary intake. A small number of studies have examined the utility of antioxidants in treating pain and preventing episodes in patients with chronic pancreatitis. ${ }^{67}$

Supplemental antioxidants are considered to be a major alternative treatment in chronic pancreatitis, but are used less often than PERT. ${ }^{54}$ A handful of trials has explored the administration of single antioxidants or as a combined formulation in patients with chronic pancreatitis or RAP. The results are mixed. Studies of allopurinol and dimethyl sulfoxide showed inconsistent results in improvement of quality of life or decreased pain intensity. ${ }^{68,69} \mathrm{~S}$-adenosyl methionine was also reported to be ineffective in a review article by Grigsby et al. ${ }^{68}$ Curcumin, a polyphenolic compound found in turmeric, was observed to decrease oxidative stress but did not exert any significant effect on pain relief. ${ }^{70}$ Some promise is seen with trials of combined antioxidant supplementation in the treatment of chronic pancreatitis. ${ }^{66}$ Two trials done with a combined antioxidant supplement containing selenium, $\beta$-carotene, $d$ - $\alpha$-tocopherol, acetate, ascorbic acid, and methionine showed improvement in quality of life and achieved a significant reduction in pain. There was also a decrease in biochemical markers of oxidative stress and improvement of antioxidant status. ${ }^{71,72}$ One of these studies also demonstrated that treatment reduced the number of episodes of acute pancreatitis. ${ }^{72}$ However, the recent completion of the ANTI-Oxidant therapy for painful
Chronic Pancreatitis Therapy Evaluation (ANTICIPATE) study concluded that patients with painful chronic pancreatitis of alcoholic origin did not experience a reduction in pain or improvement in quality of life with combined antioxidant therapy, despite a sustained increase in blood levels of the antioxidants. ${ }^{73}$ The authors mentioned that their cohort consisted of older patients (median 50 years versus 30 years in the study by Bhardwaj et al) who likely had irreversible pancreatic damage that could not be modified by antioxidant supplementation. ${ }^{72}$ These findings are similar to the results obtained by Burton et al who found that administration of antioxidants was more effective in younger patients and those with a pancreatitis etiology secondary to idiopathic and obstructive versus alcoholic pancreatitis. ${ }^{72}$ There are no studies investigating the effects of antioxidant supplementation in a younger cohort of patients in the early stages of recurrent acute pancreatitis.

EGb 761 is a standardized extract from the Gingko biloba tree, a herbal supplement utilized in traditional Chinese medicine. An animal study showed that prophylactic treatment with this extract resulted in a decrease in serum amylase and lipase levels as well as better histopathologic appearance of the pancreas. ${ }^{74}$ There was minimal parenchymal inflammation and edema on pancreatic histopathology samples. The authors concluded that attenuation of inflammation was secondary to the antioxidant effect of EGb 761.

Quercetin, naturally found in fruits and in some Chinese herbs, is a plant flavonoid reported to have anti-inflammatory and antioxidant properties. An animal study evaluating its effect on experimental pancreatitis was completed by Carvalho et al, ${ }^{75}$ who observed that mice pretreated with quercetin as an oral supplement prior to cerulein-induced pancreatitis had less biochemical and histological evidence of pancreatic inflammation. This study group concluded that quercetin can decrease inflammation in experimental acute pancreatitis via its antioxidant and anti-inflammatory properties.

Two types of tea extract, black and green, both from the Camellia sinensis plant have been reported to decrease the effects of pancreatitis in animal studies. ${ }^{76,77}$ In rats with ethanol-induced and cholecystokinin-induced pancreatitis, feeding with black tea extract allowed for improved amylase and lipase levels with increased anti-inflammatory, antioxidant, and antiapoptotic activity. ${ }^{76}$ Polyphenols from green tea were studied in mice with cerulein-induced pancreatitis. ${ }^{77}$ The study group observed that intraperitoneal administration of green tea polyphenols caused attenuation of the effect of pancreatitis through modification of inflammatory 


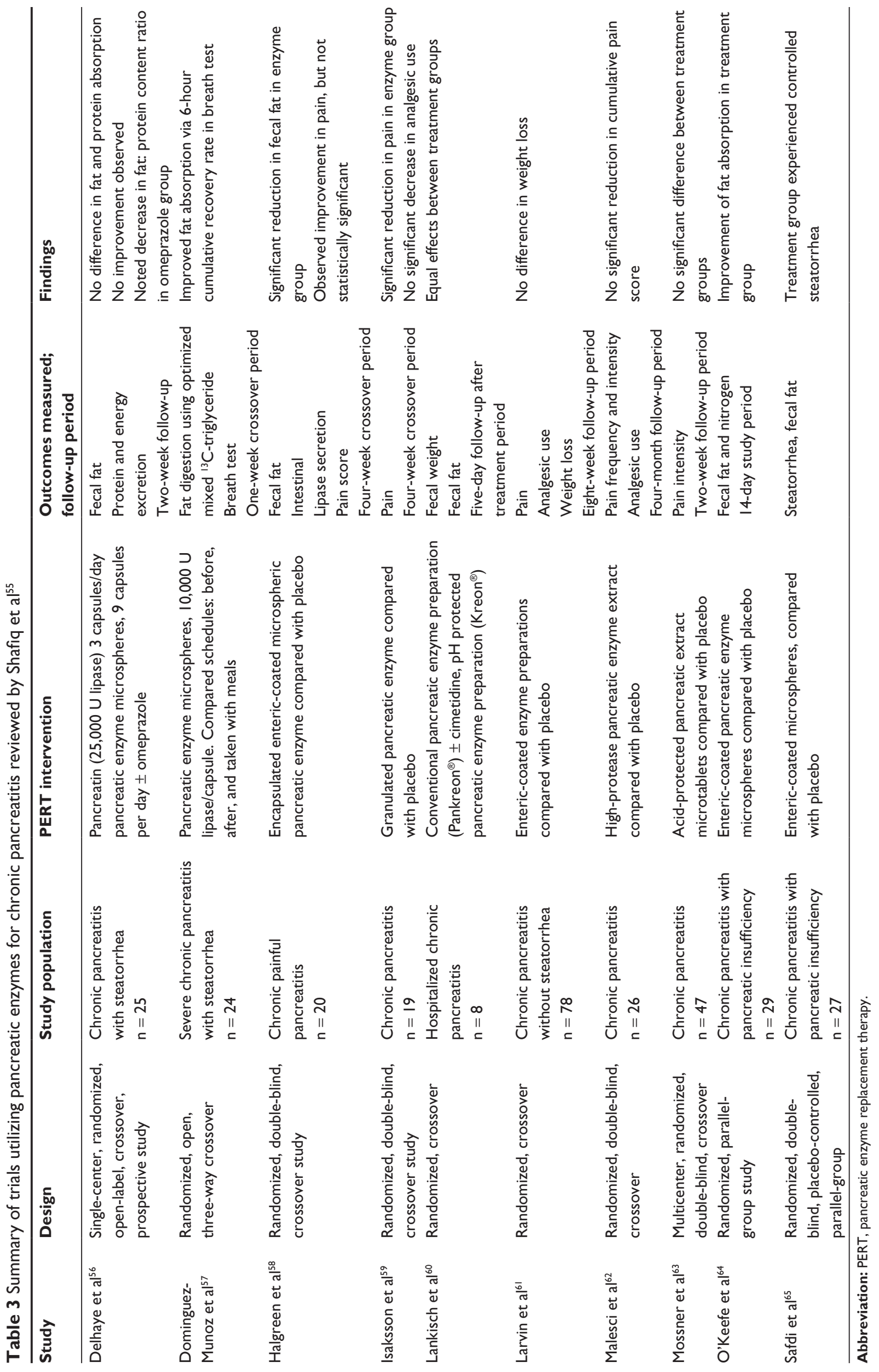


and oxidative stress pathways. Emblica officinalis, or Indian gooseberry, is a medicinal plant available in powder form. ${ }^{78}$ In animals with L-arginine-induced pancreatitis, treatment with oral doses of E. officinalis led to improvement of biomarkers of pancreatitis. Histopathology of the pancreas in animals treated with E. officinalis showed decreased destruction of acinar cells, minimal focal inflammation, and no evidence of fat necrosis. Grape seed extract has been reported to have antioxidative and anti-inflammatory effects. ${ }^{79}$ There is anecdotal evidence in affected patients that commercially available grape seed extract can reduce symptoms of chronic pancreatitis. ${ }^{80}$ Milk thistle extract acts to protect the liver from injury. ${ }^{81} \mathrm{~A}$ main component is the flavonolignan, silibinin. An animal study to determine its effect on the pancreas was completed by von Schönfeld et al. ${ }^{82}$ Pancreatic injury was induced via cyclosporine in rats that were subsequently treated with silibinin intraperitoneally. The results showed that rats treated with silibinin had restoration of amylase secretion that was initially inhibited by cyclosporine. The study concluded that silibinin may protect pancreatic exocrine cells. ${ }^{82}$ Table 4 summarizes the effects of some herbal supplements used in RAP and chronic pancreatitis. There are no large, controlled trials on the use of herbal supplements to prevent RAP in humans.

\section{Other components and functional foods}

Glycine, an inhibitory neurotransmitter and cytoprotective amino acid, has been studied in a rat model with mild cerulein-induced pancreatitis and severe taurocholateinduced pancreatitis. ${ }^{83}$ Prior to induction of pancreatitis, rats were given intravenous doses of glycine. After treatment, it was noted that rats with mild cerulein-induced pancreatitis pretreated with glycine had less pancreatic necrosis and inflammation. In rats with severe pancreatitis, there was

Table 4 Herbal supplements taken in recurrent episodes of acute pancreatitis and chronic pancreatitis

\begin{tabular}{ll}
\hline Herbal supplement & Active component; effect \\
\hline $\begin{array}{l}\text { Gingko biloba extract } \\
\text { Quercetin }\end{array}$ & $\begin{array}{l}\text { EgB76I; antioxidant } \\
\text { Plant flavonoid; antioxidant and anti- } \\
\text { inflammatory }\end{array}$ \\
$\begin{array}{l}\text { Black and green tea extract } \\
\text { (Camellia sinensis) }\end{array}$ & $\begin{array}{l}\text { Polyphenol; antioxidant, anti- } \\
\text { inflammatory and antiapoptotic }\end{array}$ \\
$\begin{array}{l}\text { Indian goose berry } \\
\text { (Emblica officinalis) }\end{array}$ & Antioxidant and anti-inflammatory \\
Grape seed extract & $\begin{array}{l}\text { Plant flavonoid and polyphenol; } \\
\text { antioxidant and anti-inflammatory } \\
\text { Milk thistle (Silybum marianum) }\end{array}$ \\
\hline
\end{tabular}

a decrease in pancreatic cytokine, myeloperoxidase, lipase, and amylase levels. Overall, glycine exhibited an attenuating effect on pancreatitis, making it a possible therapeutic component in prophylaxis.

Zinc is present in high concentrations in pancreatic juice. A population study done in India determined that patients with chronic pancreatitis have low erythrocyte zinc levels, particularly in cases of tropical pancreatitis ${ }^{84} \mathrm{~A}$ prior study showed that zinc plays an important role in maintaining the integrity of pancreatic acinar cells. Analysis of zinc-deficient rats showed significant changes in acinar cells, with a reduction in zymogen granules, and an increase in lysosomes and cytoplasmic degradation. ${ }^{85}$ Pancreatic function shows some dependence on zinc, and supplementation may contribute to maintenance of function.

Blueberries contain the polyphenol, pterostilbene, that has antioxidant and anti-inflammatory properties. ${ }^{86} \mathrm{An}$ in vitro study aimed to assess its effect on pancreatitis induced by tumor necrosis factor alpha. The study showed that acinar cells treated with pterostilbene had lower levels of inflammatory markers. ${ }^{87}$ Patients with pancreatitis may have lower levels of carotenoids, one of which is lycopene. ${ }^{88,89}$ Isolated rat pancreatic acinar cells with cerulein-induced pancreatitis showed a decrease in interleukin- 6 and reactive oxygen species after treatment with lycopene..$^{90}$ Although it is possible to increase carotenoid levels in patients with pancreatitis, it is not clear whether dietary supplementation can prevent recurrent episodes or ameliorate symptoms. ${ }^{89}$ There are no rigorous studies examining the efficacy of consumption of popular known functional foods (also known as superfoods) in preventing RAP.

\section{Conclusion}

Pancreatitis is an inflammatory condition that is clinically and economically relevant in modern medicine. Treatment is mainly supportive, and patients with chronic pancreatitis experience complications that are irreversible and have a poor prognosis. This outcome makes finding ways to prevent recurrent episodes of pancreatitis, which precede chronic changes, very important. Although nutritional advice is frequently given patients with pancreatitis, most is of questionable efficacy. The most successful interventions include abstinence from alcohol in patients with alcohol-associated pancreatitis and treatment of dyslipidemias to reduce serum triglyceride levels. Other approaches are not supported by empirical evidence. Common interventions like a low-fat diet and PERT have never been conclusively shown to alleviate symptoms or prevent recurrent episodes of pancreatitis. 
Antioxidant therapy shows promise but the data are mixed and there is no clear evidence to guide selection of antioxidants or the appropriate dose. There is a lack of investigations that include patients who are early in the course of recurrent acute pancreatitis and it is not known if that patient population would benefit from any dietary intervention. More studies are needed to find effective nutritional modifications, whether pharmacologic, dietary or behavioral, that can alter the course of acute pancreatitis.

\section{Disclosure}

The authors report no conflicts of interest in this work.

\section{References}

1. McClave SA, Snider H, Owens N, Sexton LK. Clinical nutrition in pancreatitis. Dig Dis Sci. 1997;42:2035-2044.

2. Etemad B, Whitcomb DC. Chronic pancreatitis: diagnosis, classification and new genetic developments. Gastroenterology. 2001;120: 682-707.

3. Everhart JE, Ruhl CE. Burden of digestive disease in the United States Part I: overall and upper gastrointestinal diseases. Gastroenterology. 2009; 136:376-386.

4. Shaw JHF, Wolfe RR. Glucose, fatty acid and urea kinetics in patients with severe pancreatitis: the response to substrate infusion and total parenteral nutrition. Ann Surg. 1986;204:665-672.

5. Dickerson RN, Vehe KL, Mullen JL, Feurer ID. Resting energy expenditure in patients with pancreatitis. Crit Care Med. 1991;19: 484-490.

6. Ammori BJ, Barclar GR, Larvin M, McMahon MJ. Hypocalcemia in patients with acute pancreatitis: a putative role for systemic endotoxin exposure. Pancreas. 2003;26:213-217.

7. Ryzen E, Rude RK. Low intracellular magnesium in patients with acute pancreatitis and hypocalcemia. West J Med. 1990;152:145-148.

8. McClave SA, Chang WK, Dhaliwal R, Heyland DK. Nutrition support in acute pancreatitis: a systematic review of the literature. $J$ Parenter Enteral Nutr. 2006;30:143-156.

9. American Gastroenterological Association. AGA Institute technical review on acute pancreatitis. Gastroenterology. 2007;132 2022-2044.

10. Marik PE, Zaloga GP. Early enteral nutrition in acutely ill patients: a systematic review. Crit Care Med. 2001;29:2264-2270.

11. Marik PE, Zaloga GP. Meta-analysis of parenteral nutrition versus enteral nutrition in patients with acute pancreatitis. BMJ. 2004;328: 1407-1412.

12. Yi F, Ge L, Zhao J, et al. Meta-analysis: total parenteral nutrition versus total enteral nutrition in predicted severe acute pancreatitis. Intern Med. 2012;51:523-530.

13. O'Keefe SJD. Physiological response of the human pancreas to enteral and parenteral feeding. Curr Opin Clin Nutr Metab Care. 2006;9: 622-628.

14. DiSario JA. Endoscopic approaches to enteral nutrition support. Best Pract Res Clin Gastroenterol. 2006;20:605-630.

15. Eatock FC, Chong P, Menezes N, et al. A randomized study of early nasogastric versus nasojejunal feeding in severe acute pancreatitis. Am J Gastroenterol. 2005;100:432-439.

16. Piciucchi M, Merola E, Marignani M, et al. Nasogastric or nasointestinal feeding in severe acute pancreatitis. World J Gastroenterol. 2010;16 3692-3696.

17. Petrov MS, Loveday BP, Pylypchuk RD, McIlroy K, Phillips AR, Windsor JA. Systematic review and meta-analysis of enteral nutrition formulations in acute pancreatitis. Br J Surg. 2009;96:1243-1252.
18. Mirtallo JM, Forbes A, McClave SA, Jensen GL, Waitzberg DL, Davies AR. International consensus guidelines for nutrition therapy in pancreatitis. J Parenter Enteral Nutr. 2012;36:284-291.

19. Teich N, Aghdassi A, Fischer J, et al. Optimal timing of oral refeeding in mild acute pancreatitis: results of an open randomized multicenter trial. Pancreas. 2010;39:1088-1092.

20. Abou-Assi S, Craig K, O’Keefe SJ. Hypocaloric jejunal feeding is better than total parenteral nutrition in acute pancreatitis: results of a randomized comparative study. Am J Gastroenterol. 2002;97: 2255-2262.

21. Petrov MS, van Santvoort HC, Besselink MG, Cirkel GA, Brink MA, Gooszen HG. Oral refeeding after onset of acute pancreatitis: a review of literature. Am J Gastroenterol. 2007;102:2079-2084.

22. Jacobson BC, Vander Vliet MB, Hughes MD, Maurer R, McManus K, Banks PA. A prospective, randomized trial of clear liquids versus low-fat solid diet at the initial meal in mild acute pancreatitis. Clin Gastroenterol Hepatol. 2007;5:946-951.

23. Moraes JM, Felga GE, Chebli LA, et al. A full solid diet as the initial meal in mild acute pancreatitis is safe and result in a shorter length of hospitalization: results from a prospective, randomized, controlled, double-blind clinical trial. J Clin Gastroenterol. 2010;44:517-522.

24. Siriwardena AK, Mason JM, Balachandra S, et al. Randomised, double blind, placebo controlled trial of intravenous antioxidant (n-acetylcysteine, selenium, vitamin C) therapy in severe acute pancreatitis. Gut. 2007;56:1439-1444.

25. Hackert T, Werner J. Antioxidant therapy in acute pancreatitis: experimental and clinical evidence. Antioxid Redox Signal. 2011;15: 2767-2777.

26. Oláh A, Belágyi T, Issekutz A, Gamal ME, Bengmark S. Randomized clinical trial of specific lactobacillus and fibre supplement to early enteral nutrition in patients with acute pancreatitis. Br J Surg. 2002;89:1103-1107.

27. Besselink MG, van Santvoort HC, Buskens E, et al. Probiotic prophylaxis in predicted severe acute pancreatitis: a randomised, double-blind, placebo-controlled trial. Lancet. 2008;371:651-659.

28. Pearce CB, Sadek SA, Walters AM, et al. A double-blind, randomised, controlled trial to study the effects of an enteral feed supplement with glutamine, arginine and omega- 3 fatty acid in predicted acute severe pancreatitis. JOP. 2006;7:361-371.

29. Yadav D, Timmons L, Benson JT, Dierkhising RA, Chari ST. Incidence, prevalence, and survival of chronic pancreatitis: a population-based study. Am J Gastroenterol. 2011;106:2192-2199.

30. Yadav D. Recent advances in the epidemiology of alcoholic pancreatitis. Curr Gastroenterol Rep. 2011;13:157-165.

31. Nordback I, Pelli H, Lappalainen-Lehto R, Järvinen S, Räty S, Sand J. The recurrence of acute alcohol-associated pancreatitis can be reduced: a randomized controlled trial. Gastroenterology. 2009;136:848-855.

32. Ammann RW, Raimondi S, Maisonneuve P, Mullhaupt B. Is obesity an additional risk factor for alcoholic chronic pancreatitis? Pancreatology. 2010;10:47-53.

33. Martínez J, Sánchez-Payá J, Palazón JM, Suazo-Barahona J, RoblesDíaz G, Pérez-Mateo M. Is obesity a risk factor in acute pancreatitis? A meta-analysis. Pancreatology. 2004;4:42-48.

34. Navina S, Acharya C, DeLany JP, et al. Lipotoxicity causes multisystem organ failure and exacerbates acute pancreatitis in obesity. Sci Transl Med. 2011;3:107-110.

35. Abu Hilal M, Armstrong T. The impact of obesity on the course and outcome of acute pancreatitis. Obes Surg. 2008;18:326-328.

36. Frossard JL, Lescuyer P, Pastor CM. Experimental evidence of obesity as a risk factor for severe acute pancreatitis. World $J$ Gastroenterol. 2009; 15:5260-5265.

37. Yadav D, Pitchumoni CS. Issues in hyperlipidemic pancreatitis. J Clin Gastroenterol. 2003;36:54-62.

38. Sandhu S, Al-Sarraf A, Taraboanta C, Frohlich J, Francis GA. Incidence of pancreatitis, secondary causes, and treatment of patients referred to a specialty lipid clinic with severe hypertriglyceridemia: a retrospective cohort study. Lipids Health Dis. 2011;10:157-164. 
39. Anderson F, Thomson SR, Clarke DL, Buccimazza I. Dyslipidaemic pancreatitis clinical assessment and analysis of disease severity and outcomes. Pancreatology. 2009;9:252-257.

40. Bugdaci MS, Sokmen M, Zuhur SS, Altuntas Y. Lipid profile changes and importance of low serum $\alpha$-lipoprotein fraction (high-density lipoprotein) in cases with acute pancreatitis. Pancreas. 2011;40: 1241-1244.

41. Lai SW, Muo CH, Liao KF, Sung FC, Chen PC. Risk of acute pancreatitis in type 2 diabetes and risk reduction on anti-diabetic drugs: a population-based cohort study in Taiwan. Am J Gastroenterol. 2011;106:1697-1704.

42. Girman CJ, Kou TD, Cai B, et al. Patients with type 2 diabetes mellitus have higher risk for acute pancreatitis compared with those without diabetes. Diabetes Obes Metab. 2010;12:766-771.

43. Gonzalez-Perez A, Schlienger RG, Rodríguez LA. Acute pancreatitis in association with type 2 diabetes and antidiabetic drugs: a populationbased cohort study. Diabetes Care. 2010;33:2580-2585.

44. Noel RA, Braun DK, Patterson RE, Bloomgren GL. Increased risk of acute pancreatitis and biliary disease observed in patients with type 2 diabetes: a retrospective cohort study. Diabetes Care. 2009;32: 834-838.

45. Keller J, Layer P. Human pancreatic exocrine response to nutrients in health and disease. Gut. 2005;54 Suppl 6:vi1-vi28.

46. Roncal-Jimenez CA, Lanaspa MA, Rivard CJ, et al. Sucrose induces fatty liver and pancreatic inflammation in male breeder rats independent of excess energy intake. Metabolism. 2011;60:1259-1270.

47. Al-Azzawi HH, Wade TE, Swartz-Basile DA, Wang S, Pitt HA, Zyromski NJ. Acute pancreatitis in obesity: adipokines and dietary fish oil. Dig Dis Sci. 2011;56:2318-2325.

48. Gastaminza G, Bernaola G, Camino ME. Acute pancreatitis caused by allergy to kiwi fruit. Allergy. 1998;53:1104-1105.

49. Matteo A, Sarles H. Is food allergy a cause of acute pancreatitis? Pancreas. 1990;5:234-237.

50. Suzuki S, Homma T, Kurokawa M, et al. Eosinophilic gastroenteritis due to cow's milk allergy presenting with acute pancreatitis. Int Arch Allergy Immunol. 2012;158 Suppl 1:75-82.

51. Patel RS, Johlin FC Jr, Murray JA. Celiac disease and recurrent pancreatitis. Gastrointest Endosc. 1999;50:823-827.

52. Lee KT, Sheen PC. Study of lysosomal changes in rat pancreas after ingesting monosodium L-glutamate. Pancreas. 1994;9:304-308.

53. Leshchenko IV, Shevchuk VH, Falalieieva TM, Beregova TV. The influence of long-term monosodium glutamate feeding on the structure of rats pancreas. Fiziol Zh. 2012;58:59-65. Ukrainian.

54. Burton F, Alkaade S, Collins D, et al. Use and perceived effectiveness of non-analgesic medical therapies for chronic pancreatitis in the United States. Aliment Pharmacol Ther. 2011;33:149-159.

55. Shafiq N, Rana S, Bhasin D, et al. Pancreatic enzymes for chronic pancreatitis. Cochrane Database Syst Rev. 2009;4:CD006302.

56. Delhaye M, Meuris S, Gohimont AC, Buedts K, Cremer M. Comparative evaluation of a high lipase pancreatic enzyme preparation and a standard pancreatic supplement for treating exocrine pancreatic insufficiency in chronic pancreatitis. Eur J Gastroenterol Hepatol. 1996;8:699-703.

57. Dominguez-Munoz JE, Iglesias-Garcia J, Iglesias-Rey M, Figueiras A, Vilarino-Insua M. Effect of the administration schedule on the therapeutic efficacy of oral pancreatic enzyme supplements in patients with exocrine pancreatic insufficiency: a randomized, three-way crossover study. Aliment Pharmacol Ther. 2005;21:993-1000.

58. Halgreen H, Pederson NT, Worning H. Symptomatic effect of pancreatic enzyme therapy in patients with chronic pancreatitis. Scand J Gastroenterol. 1986;21:104-108.

59. Isaksson G, Ihse I. Pain reduction by an oral pancreatic enzyme preparation in chronic pancreatitis. Dig Dis Sci. 1983;28:97-102.

60. Lankisch PG, Lembcke B, Goke B, Creutzfeldt W. Therapy of pancreatogenic steatorrhea: does acid protection of pancreatic enzymes offer any advantage? Z Gastroenterol. 1986;24:753-757.
61. Larvin M, McMahon MJ, Thomas WEG, Puntis MCA. Creon (enteric coated pancreatin microspheres) for the treatment of pain in chronic pancreatitis: a double blind randomized placebo-controled cross-over study. Gastroenterology. 1991;100:A283.

62. Malesci A, Gaia E, Fioretta A, et al. No effect of long term treatment with pancreatic extract on recurrent abdominal pain in patients with chronic pancreatitis. Scand J Gastroenterol. 1995;30:392-398.

63. Mossner J, Secknus R, Mayer J, Niedeau C, Adler G. Treatment of pain with pancreatic extracts in chronic pancreatitis: results of a prospective placebo-controlled multicenter trial. Digestion. 1992;53:54-66.

64. O'Keefe SJ, Cariem AK, Levy M. The exacerbation of pancreatic endocrine dysfunction by potent pancreatic exocrine supplements in patients with chronic pancreatitis. J Clin Gastroenterol. 2001;32: 319-323.

65. Safdi M, Bekal PK, Martin S, Saeed ZA, Burton F, Toskes PP. The effects of oral pancreatic enzymes (Creon 10 capsule) on steatorrhea: a multi-center, placebo-controlled, parallel group trial in subjects with chronic pancreatitis. Pancreas. 2006;33:156-162.

66. Grigsby B, Rodriguez-Rilo H, Khan K. Antioxidants and chronic pancreatitis: theory of oxidative stress and trials of antioxidant therapy. Dig Dis Sci. 2012;57:835-841.

67. Braganza JM, Dormandy TL. Micronutrient therapy for chronic pancreatitis: rationale and impact. JOP. 2010;11:99-112.

68. Salim AS. Role of oxygen-derived free radical scavengers in the treatment of recurrent pain produced by chronic pancreatitis: a new approach. Arch Surg. 1991;126:1109-1114.

69. Banks PA, Hughes M, Ferrante M, Noordhoek EC, Ramagopal V, Slivka A. Does allopurinol reduce pain of chronic pancreatitis? Int $J$ Pancreatol. 1997;22:171-176.

70. Durgaprasad S, Pai CG, Vasanthkumar, Alvres JF, Namitha S. A pilot study of the antioxidant effect of curcumin in tropical pancreatitis. Indian J Med Res. 2005;122:315-318.

71. Kirk GR, White JS, McKie L, et al. Combined antioxidant therapy reduces pain and improves quality of life in chronic pancreatitis. J Gastrointest Surg. 2006;10:499-503.

72. Bhardwaj P, Garg PK, Maulik SK, Saraya A, Tandon RK, Acharya SK. A randomized controlled trial of antioxidant supplementation for pain relief in patients with chronic pancreatitis. Gastroenterology. 2009;136:149-159.

73. Siriwardena AK, Mason JM, Sheen AJ, Makin AJ, Shah NS. Antioxidant therapy does not reduce pain in patients with chronic pancreatitis: the ANTICIPATE study. Gastroenterology. 2012;143:655-663.

74. Zeybek N, Gorgulu S, Yagci G, et al. The effects of gingko biloba extract (EGb 761) on experimental acute pancreatitis. J Surg Res. 2003;115: 286-293.

75. Carvalho KM, Morais TC, de Melo TS, et al. The natural flavonoid quercetin ameliorates cerulein-induced acute pancreatitis in mice. Biol Pharm Bull. 2010;33:1534-1539.

76. Das D, Mukherjee S, Das AS, Mukherjee M, Mitra C. Aqueous extract of black tea (camellia sinensis) prevents ethanol + cholecystokinininduced pancreatitis in a rat model. Life Sci. 2006;78:2194-2203.

77. Babu BI, Malleo G, Genovese T, et al. Green tea polyphenols ameliorate pancreatic injury in cerulein-induced murine acute pancreatitis. Pancreas. 2009;38:954-967.

78. Sidhu S, Pandhi P, Malhotra S, Vaiphei K, Khanduja KL. Beneficial effects of emblica officinalis in L-arginine-induced acute pancreatitis in rats. $J$ Med Food. 2011;14:147-155.

79. Nassiri-Asl M, Hosseinzadeh H. Review of the pharmacological effects of vitis vinifera (grape) and its bioactive compounds. Phytother Res. 2009;23:1197-1204.

80. Banerjee B, Bagchi D. Beneficial effects of a novel IH636 grape seed proanthocyanidin extract in the treatment of chronic pancreatitis. Digestion. 2001;63:203-206.

81. Tamayo C, Diamond S. Review of clinical trials evaluating safety and efficacy of milk thistle (silybum marianum [L.] gaertn.). Integr Cancer Ther. 2007;6:146-157. 
82. von Schönfeld J, Weisbrod B, Müller MK. Silibinin, a plant extract with antioxidant and membrane stabilizing properties, protects exocrine pancreas from cyclosporin A toxicity. Cell Mol Life Sci. 1997;53: 917-920.

83. Ceyhan GO, Timm AK, Bergmann F, et al. Prophylactic glycine administration attenuates pancreatic damage and inflammation in experimental acute pancreatitis. Pancreatology. 2011;11:57-67.

84. Girish BN, Rajesh G, Vaidyanathan K, Balakrishnan V. Zinc status in chronic pancreatitis and its relationship with exocrine and endocrine insufficiency. JOP. 2009;10:651-656.

85. Koo SI, Turk DE. Effect of zinc deficiency on the ultrastructure of the pancreatic acinar cell and intestinal epithelium in the rat. J Nutr. 1977; 107:896-908.
86. [No authors listed]. Pterostilbene. Monograph. Altern Med Rev. 2010 15:159-163.

87. McCormack D, McDonald D, McFadden D. Pterostilbene ameliorates tumor necrosis factor alpha-induced pancreatitis in vitro. J Surg Res. 2012;178:28-32.

88. Kim H. Inhibitory mechanism of lycopene on cytokine expression in experimental pancreatitis. Ann N Y Acad Sci. 2011;1229:99-102.

89. Quilliot D, Forbes A, Dubois F, Gueant JL, Ziegler O. Carotenoid deficiency in chronic pancreatitis: the effect of an increase in tomato consumption. Eur J Clin Nutr. 2011;65:262-268.

90. Kang M, Park KS, Seo JY, Kim H. Lycopene inhibits IL-6 expression in cerulein-stimulated pancreatic acinar cells. Genes Nutr. 2011;6: $117-123$.
Nutrition and Dietary Supplements

\section{Publish your work in this journal}

Nutrition and Dietary Supplements is an international, peer-reviewed, open access journal focusing on research into nutritional requirements in health and disease, impact on metabolism and the identification and optimal use of dietary strategies and supplements necessary for normal growth and development. The journal welcomes papers covering

\section{Dovepress}

original research, basic science, clinical \& epidemiological studies, reviews and evaluations, guidelines, expert opinion and commentary, case reports and extended reports. The manuscript management system is completely online and includes a very quick and fair peer-review system, which is all easy to use.

Submit your manuscript here: http://www.dovepress.com/nutrition-and-dietary-supplements-journal 